\title{
A harmonic maps approach to fluid flows
}

\author{
Olivia Constantin'1 • María J. Martín²
}

Received: 2 December 2015 / Revised: 6 April 2016 / Published online: 4 June 2016 (C) The Author(s) 2016. This article is published with open access at Springerlink.com

\begin{abstract}
We obtain a complete solution to the problem of classifying all twodimensional ideal fluid flows with harmonic Lagrangian labelling maps; thus, we explicitly provide all solutions, with the specified structural property, to the incompressible two-dimensional Euler equations (in Lagrangian variables).
\end{abstract}

Mathematics Subject Classification 76B03 · 35Q31 · 76M40

\section{Introduction}

Within the realm of fluid dynamics, the most complete description of a flow is attained within the Lagrangian framework. Qualitative studies of fluids are concerned with perturbations of explicitly known flows. Therefore non-trivial explicit solutions that

Dedicated to Professor Peter Duren on the occasion of his 80th birthday.

Communicated by Y. Giga.

The Olivia Constantin was supported by FWF Project P 24986-N25. The María J. Martín was supported in part by Academy of Finland Grant 268009 and by Spanish MINECO/FEDER Research Project MTM2015-65792-P.

Olivia Constantin

olivia.constantin@univie.ac.at

María J. Martín

maria.martin@uef.fi

1 Faculty of Mathematics, University of Vienna, Oskar-Morgenstern-Platz 1, 1090 Vienna, Austria

2 Department of Physics and Mathematics, University of Eastern Finland, P.O. Box 111, 80101 Joensuu, Finland 
capture important aspects of the physical reality are of great importance [3]. Several publications in the applied mathematics, engineering and physics research literature (see $[6,7,10-12,15,18]$ and references therein) exploited a remarkable feature shared by some celebrated explicit solutions to the two-dimensional incompressible Euler equations, in Lagrangian variables (such as Kirchhoff's elliptical vortex [16] found in 1876, Gerstner's flow [9] found in 1809 and re-discovered in 1863 by Rankine [19], and the Ptolemaic vortices found in 1984 by Abrashkin and Yakubovich [1]), namely that in all of them the labelling map is harmonic at all times.

Recently, in [2], the authors proposed a complex analysis approach aimed at classifying all such flows. While new explicit solutions were obtained, the exhaustion of all possibilities was reduced to an explicit nonlinear ordinary differential system in $\mathbb{C}^{4}$. Solving this system in full generality proved elusive so far.

We propose a different approach that provides a complete solution to the original problem of finding all flows with harmonic labelling maps. Our approach is based on ideas from the theory of harmonic mappings, more precisely, on the fact that it is possible to characterize the relationship between planar harmonic maps having the same Jacobian - a property of the labelling maps that is a consequence of the equation of mass conservation, expressed in Lagrangian variables. Apart from achieving the full picture, our considerations provide an illustration of the deep links between the fields of complex analysis and fluid mechanics.

\section{The governing equations}

The Eulerian description of the two-dimensional motion of an ideal homogeneous fluid is obtained by imposing the law of mass conservation

$$
u_{x}+v_{y}=0
$$

and Euler's equation of motion

$$
\left\{\begin{array}{l}
u_{t}+u u_{x}+v u_{y}=-P_{x} \\
v_{t}+u v_{x}+v v_{y}=-P_{y}
\end{array}\right.
$$

where $(u(t, x, y), v(t, x, y))$ is the velocity field in the time and space variables $(t, x, y)$ and the scalar function $P(t, x, y)$ represents the pressure. Since the reference density in hydrodynamics is $1 \mathrm{~g} / \mathrm{cm}^{3}$, we normalize the constant fluid density to 1.

The most complete flow representation is provided in (material) Lagrangian coordinates, in which one describes the motion of all fluid particles. For a given velocity field $(u(t, x, y), v(t, x, y))$, the motion of the individual particles $(x(t), y(t))$ is obtained by integrating the system of ordinary differential equations

$$
\left\{\begin{array}{l}
x^{\prime}(t)=u(t, x, y), \\
y^{\prime}(t)=v(t, x, y),
\end{array}\right.
$$


whereas the knowledge of the particle path $t \mapsto(x(t), y(t))$ provides by differentiation with respect to $t$ the velocity field at time $t$ and at the location $(x(t), y(t))$.

Starting with a simply connected domain $\Omega_{0}$, representing the labelling domain, each label $(a, b) \in \Omega_{0}$ identifies by means of the injective map

$$
(a, b) \mapsto(x(t ; a, b), y(t ; a, b))
$$

the evolution in time of a specific particle, the fluid domain at time $t, \Omega(t)$, being the image of $\Omega_{0}$ under the map (3). To write the governing equations in Lagrangian coordinates, we use the following relations:

$$
\left\{\begin{array}{l}
\frac{\partial}{\partial a}=x_{a} \frac{\partial}{\partial x}+y_{a} \frac{\partial}{\partial y}, \\
\frac{\partial}{\partial b}=x_{b} \frac{\partial}{\partial x}+y_{b} \frac{\partial}{\partial y}
\end{array}\right.
$$

and

$$
\left\{\begin{array}{l}
\frac{\partial}{\partial x}=\frac{1}{J}\left(y_{b} \frac{\partial}{\partial a}-y_{a} \frac{\partial}{\partial b}\right), \\
\frac{\partial}{\partial y}=\frac{1}{J}\left(x_{a} \frac{\partial}{\partial b}-x_{b} \frac{\partial}{\partial a}\right),
\end{array}\right.
$$

where $J$ is the Jacobian of the transformation given by

$$
J=\left|\frac{\partial(x, y)}{\partial(a, b)}\right|=x_{a} y_{b}-y_{a} x_{b} \neq 0 .
$$

The local injectivity of the transformation between the Eulerian and Lagrangian coordinates is expressed by (5). In view of (4) and

$$
\left\{\begin{array}{l}
u(t, x, y)=\frac{\partial}{\partial t} x(t ; a, b), \\
v(t, x, y)=\frac{\partial}{\partial t} y(t ; a, b),
\end{array}\right.
$$

the equation of mass conservation (1) takes the form

$$
0=u_{x}+v_{y}=\frac{y_{b} x_{a t}-y_{a} x_{b t}+x_{a} y_{b t}-x_{b} y_{a t}}{J}=\frac{J_{t}}{J}
$$

in Lagrangian coordinates, that is,

$$
J_{t}=0 \text {. }
$$

On the other hand, from (6) we get

$$
\begin{aligned}
& x_{t t}=u_{t}+u_{x} x_{t}+u_{y} y_{t}=u_{t}+u u_{x}+v u_{y}, \\
& y_{t t}=v_{t}+u v_{x}+v v_{y},
\end{aligned}
$$


so that the Euler equation (2), in Lagrangian variables, becomes

$$
\left\{\begin{array}{l}
x_{t t}=-\frac{y_{b} P_{a}-y_{a} P_{b}}{J}, \\
y_{t t}=-\frac{x_{a} P_{b}-x_{b} P_{a}}{J} .
\end{array}\right.
$$

Since the Jacobian of the above system does not vanish, due to (5), we can solve for the gradient of $P$ in the label space, obtaining

$$
\left\{\begin{array}{l}
P_{a}=-x_{a} x_{t t}-y_{a} y_{t t} \\
P_{b}=-x_{b} x_{t t}-y_{b} y_{t t}
\end{array}\right.
$$

in $\Omega_{0}$. The domain $\Omega_{0}$ being simply connected, the above system is equivalent to the compatibility condition $P_{a b}=P_{b a}$, that is,

$$
x_{a} x_{b t t}+y_{a} y_{b t t}=x_{b} x_{a t t}+y_{b} y_{a t t}
$$

or, equivalently,

$$
\left(x_{a} x_{b t}+y_{a} y_{b t}-x_{b} x_{a t}-y_{b} y_{a t}\right)_{t}=0 .
$$

These considerations show that, in Lagrangian coordinates, the governing equations are equivalent to (7) and (8), under the constraint that, at any instant $t$, the map (3) is a global diffeomorphism from the label domain $\Omega_{0}$ to the fluid domain $\Omega(t)$.

\section{Harmonic labellings}

As already mentioned in Introduction, the common structural property of the known explicit solutions to the governing equations (Kirchhoff's vortex [16], Gerstner's wave [9], the Ptolemaic solutions [1]) is that the labelling map (3) is harmonic at every fixed time $t$. Our aim is to explicitly find all solutions having this property. Most ideas in this section are inspired by [2] and are included for the sake of completeness. Since from now on our methods will rely exclusively on complex analysis, it is convenient to adapt the notation accordingly. Therefore we look for solutions to (7) and (8) having the form

$$
x(t ; a, b)+i y(t ; a, b)=F(t, z)+\overline{G(t, z)}, \quad z=a+i b,
$$

with $z \mapsto F(t, z)$ and $z \mapsto G(t, z)$ analytic in the simply connected domain $\Omega_{0} \subset \mathbb{C}$, at every time $t$. Recall that $\frac{\partial F}{\partial \bar{z}}=0$ characterizes analyticity and that

$$
\frac{\partial}{\partial a}=\frac{\partial}{\partial z}+\frac{\partial}{\partial \bar{z}}, \quad \frac{\partial}{\partial b}=i\left(\frac{\partial}{\partial z}-\frac{\partial}{\partial \bar{z}}\right), \quad \frac{\partial \bar{f}}{\partial \bar{z}}=\frac{\overline{\partial f}}{\partial z} .
$$


The Jacobian of the harmonic map (9) is $J=\left|F^{\prime}\right|^{2}-\left|G^{\prime}\right|^{2}$. Therefore the equation of mass conservation (7) can be re-written as $\left(F^{\prime} \overline{F^{\prime}}-\overline{G^{\prime}} G^{\prime}\right)_{t}=0$, that is,

$$
\operatorname{Re}\left(F_{t}^{\prime} \overline{F^{\prime}}-G^{\prime} \overline{G_{t}^{\prime}}\right)=0 .
$$

Relation (10) together with (9) yield

$$
\begin{array}{ll}
x_{a}=\frac{F^{\prime}+G^{\prime}+\overline{F^{\prime}}+\overline{G^{\prime}}}{2}, & x_{b}=i \frac{F^{\prime}+G^{\prime}-\overline{F^{\prime}}-\overline{G^{\prime}}}{2}, \\
y_{a}=\frac{F^{\prime}-G^{\prime}-\overline{F^{\prime}}+\overline{G^{\prime}}}{2 i}, & y_{b}=\frac{F^{\prime}-G^{\prime}+\overline{F^{\prime}}-\overline{G^{\prime}}}{2} .
\end{array}
$$

A lengthy but straightforward calculation using the above relations shows that (8) is equivalent to

$$
\operatorname{Im}\left(F_{t}^{\prime} \overline{F^{\prime}}-G^{\prime} \overline{G_{t}^{\prime}}\right)_{t}=0
$$

In view of (11) and (12), the governing equations reduce to the single equation

$$
F_{t}^{\prime} \overline{F^{\prime}}-G^{\prime} \overline{G_{t}^{\prime}}=i v(z, \bar{z})
$$

for some real-valued function $v$.

Remark 1 Using the notation $F_{0}:=F(0, \cdot), G_{0}:=G(0, \cdot)$, the equation of mass conservation expressed as $J_{t}=0$ means that the Jacobian of the labelling map is constant in time, and hence it is given by $J=\left|F_{0}^{\prime}\right|^{2}-\left|G_{0}^{\prime}\right|^{2}$. Since $\Omega_{0}$ is simply connected and $J$ does not vanish in $\Omega_{0}$, we deduce that we either have $J>0$ (i.e. $F_{0}+\overline{G_{0}}$ is sense preserving) or $J<0$ (i.e. $F_{0}+\overline{G_{0}}$ is sense reversing) throughout $\Omega_{0}$. From now on we shall assume without loss of generality that $F_{0}+\overline{G_{0}}$ is sense preserving. Indeed, if $F_{0}+\overline{G_{0}}$ is sense reversing, we can replace the label domain by $\overline{\Omega_{0}}$, and, in this case, the labelling map at time $t=0$ becomes $F_{0}(\bar{z})+\overline{G_{0}(\bar{z})}$, which is sense preserving.

An equivalent condition to the fact that $F_{0}+\overline{G_{0}}$ is sense preserving in the simply connected domain $\Omega_{0}$ is that $F_{0}$ is locally univalent (thus $F_{0}^{\prime} \neq 0$ in $\Omega_{0}$ ) and the (second complex) dilatation $\omega$ of $F_{0}+\overline{G_{0}}$, defined by $\omega=G_{0}^{\prime} / F_{0}^{\prime}$, is an analytic map into the open unit disk.

Since the Jacobian of the harmonic maps $F(t, z)+\overline{G(t, z)}$, defined for $t \geq 0$ and $z \in \Omega_{0}$, that satisfy (13) is independent of time, in order to find all solutions it is natural to start by looking for a characterization of the relationship between two harmonic maps with equal Jacobians.

\section{Harmonic functions with equal Jacobian}

We now find the relationship between two harmonic maps with equal Jacobians. Our approach is inspired by the proof of [4, Theorem 3] (see also the considerations made in [14]). 
We start by proving the following lemma that might have some independent interest and will be used later on.

Lemma 1 Let $\varphi$ and $\psi$ be two analytic functions in a simply connected domain $\Omega$, with $\varphi \not \equiv 0$. Then

$$
|\varphi|^{2}=r|\psi|^{2}+s
$$

in $\Omega$ for some real numbers $r$ and $s$ different from zero if and only if there exist two complex constants $c_{1}$ and $c_{2}$ with $\left|c_{1}\right|^{2}=r\left|c_{2}\right|^{2}+s$ such that $\varphi \equiv c_{1}$ and $\psi \equiv c_{2}$.

Proof Note that for (14) to hold, it is necessary that $r|\psi(z)|^{2}+s \geq 0$ for all $z \in \Omega$. If $r|\psi|^{2}+s \equiv 0$, then a direct application of the open mapping theorem for analytic functions gives us the desired result.

Assume now that $r|\psi|^{2}+s$ is not identically zero. Then, there exists a disk $D=$ $D\left(z_{0}, R\right)$ centered at some $z_{0} \in \Omega$ and radius $R>0$ such that $D \subset \Omega$ and $r|\psi(z)|^{2}+$ $s>0$ for all $z \in D$. The forthcoming analysis will be done in this disk.

We take logarithms in (14) to get

$$
\log |\varphi|^{2}=\log \left(r|\psi|^{2}+s\right)
$$

Now, the function of the left-hand side in (15) is harmonic so that the one on the right-hand side must be harmonic as well. The Laplacian of this latter function equals

$$
\Delta \log \left(r|\psi|^{2}+s\right)=4 r s \frac{\left|\psi^{\prime}\right|^{2}}{\left(r|\psi|^{2}+s\right)^{2}}
$$

so that $\psi^{\prime}$ is identically zero in $D$ and hence there exists a non-zero constant $c_{2} \in \mathbb{C}$ such that $\psi \equiv c_{2}$. Bearing in mind (14), we get that $\varphi$ equals a constant $c_{1}$ too, with $\left|c_{1}\right|^{2}=r\left|c_{2}\right|^{2}+s$. A direct application of the identity principle for analytic functions completes the proof.

\subsection{The case of linear dependence}

We first treat the easier case when the harmonic mapping $F+\bar{G}$ is such that $F^{\prime}$ and $G^{\prime}$ are linearly dependent.

Theorem 1 Let $F_{1}+\overline{G_{1}}$ be a (sense preserving) harmonic map in a simply connected domain $\Omega \subset \mathbb{C}$. Assume further that $G_{1}^{\prime}=\lambda F_{1}^{\prime}$ where $\lambda \in \mathbb{C}$. If $F_{2}+\overline{G_{2}}$ is a harmonic map in $\Omega$ whose Jacobian equals that of $F_{1}+\overline{G_{1}}$, that is,

$$
\left|F_{1}^{\prime}\right|^{2}-\left|G_{1}^{\prime}\right|^{2}=\left|F_{2}^{\prime}\right|^{2}-\left|G_{2}^{\prime}\right|^{2}
$$

then there exist constants $\alpha, \beta \in \mathbb{C}$ such that $F_{2}^{\prime}=\alpha F_{1}^{\prime}$ and $G_{2}^{\prime}=\beta F_{1}^{\prime}$, with $|\alpha|^{2}-|\beta|^{2}=1-|\lambda|^{2}>0$. Moreover, if $F_{1}+\overline{G_{1}}$ is univalent in $\Omega$, then $F_{2}+\overline{G_{2}}$ is univalent in $\Omega$. 
Proof Let us first notice that $F_{1}^{\prime}$ has no zeros in $\Omega$ since the Jacobian $J_{1}$ of $F_{1}+\overline{G_{1}}$ is strictly positive. Dividing by $\left|F_{1}^{\prime}\right|^{2}$ in (16), we get

$$
1-|\lambda|^{2}=\left|\frac{F_{2}^{\prime}}{F_{1}^{\prime}}\right|^{2}-\left|\frac{G_{2}^{\prime}}{F_{1}^{\prime}}\right|^{2} .
$$

We can now apply Lemma 1 to deduce that there exist $\alpha, \beta \in \mathbb{C}$ such that $F_{2}^{\prime}=\alpha F_{1}^{\prime}$ and $G_{2}^{\prime}=\beta F_{1}^{\prime}$, with $1-|\lambda|^{2}=|\alpha|^{2}-|\beta|^{2}$. In particular, $|\alpha|^{2}-|\beta|^{2}>0$ since $J_{1}>0$.

It remains to show that univalence is preserved. If $F_{1}+\overline{G_{1}}$ is univalent, then, due to the special form of $G_{1}$, we have that $F_{1}$ is also univalent. Assume there exist $z, w \in \Omega$ with $F_{2}(z)+\overline{G_{2}(z)}=F_{2}(w)+\overline{G_{2}(w)}$. Since $F_{2}+\overline{G_{2}}=\alpha F_{1}+\overline{\beta F_{1}}+\gamma$ for some constant $\gamma \in \mathbb{C}$, the last equality yields $\alpha\left(F_{1}(z)-F_{1}(w)\right)=-\overline{\beta\left(F_{1}(z)-F_{1}(w)\right)}$. Taking the modulus on both sides, we get $F_{1}(z)-F_{1}(w)=0$, since $|\alpha| \neq|\beta|$. The univalence of $F_{1}$ forces $z=w$, thus proving the claim.

\subsection{Linearly independent case}

We now investigate the generic setting.

Theorem 2 Let $F_{1}+\overline{G_{1}}$ and $F_{2}+\overline{G_{2}}$ be two harmonic mappings on a simply connected domain $\Omega$, with Jacobians $J_{1}=\left|F_{1}^{\prime}\right|^{2}-\left|G_{1}^{\prime}\right|^{2}$ and $J_{2}=\left|F_{2}^{\prime}\right|^{2}-\left|G_{2}^{\prime}\right|^{2}$, respectively. Assume that $F_{1}^{\prime}$ and $G_{1}^{\prime}$ are linearly independent. If $J_{1}=J_{2}>0$, then there exist two complex constants $\alpha, \beta$ with $|\alpha|^{2}=1+|\beta|^{2}$ and a real number $\xi$ such that

$$
\left(\begin{array}{l}
F_{2}^{\prime} \\
G_{2}^{\prime}
\end{array}\right)=\left(\begin{array}{ll}
\alpha & \beta \\
\bar{\beta} & \bar{\alpha}
\end{array}\right)\left(\begin{array}{ll}
1 & 0 \\
0 & e^{i \xi}
\end{array}\right)\left(\begin{array}{l}
F_{1}^{\prime} \\
G_{1}^{\prime}
\end{array}\right) .
$$

Proof Since, by assumption, $\left|F_{1}^{\prime}\right|>0$, we may denote $\varphi=F_{2}^{\prime} / F_{1}^{\prime}$. Also, the dilatation $\omega_{1}=G_{1}^{\prime} / F_{1}^{\prime}$ of $F_{1}+\overline{G_{1}}$ is not constant. Since $J_{1}=J_{2}$, we have

$$
1-\left|\omega_{1}\right|^{2}=|\varphi|^{2}-|\psi|^{2}
$$

where $\psi=\omega_{2} \varphi=G_{2}^{\prime} / F_{1}^{\prime}$. We rewrite this as

$$
1+|\psi|^{2}=\left|\omega_{1}\right|^{2}+|\varphi|^{2}
$$

Note that if $\varphi$ equals a constant $k$ (necessarily different from zero), then the previous equation can be written as

$$
1+|\psi|^{2}=\left|\omega_{1}\right|^{2}+|k|^{2}
$$

If $|k|=1$, then $\left|F_{2}^{\prime}\right|=\left|F_{1}^{\prime}\right|$ and the above relation implies $\left|G_{1}^{\prime}\right|=\left|G_{2}^{\prime}\right|$. In view of the open mapping theorem we must have $F_{2}^{\prime}=e^{i s_{1}} F_{1}^{\prime}$ and $G_{2}^{\prime}=e^{i s_{2}} G_{1}^{\prime}$ for some real constants $s_{1}$ and $s_{2}$, and then (17) holds with $\beta=0, \alpha=e^{i s_{1}}$, and $\xi=s_{1}+s_{2}$. 
If $|k| \neq 1$, then by Lemma 1 we have that $\omega_{1}$ is constant, which contradicts our assumption. Thus, we can assume that $\varphi$ is not constant, so that its derivative is not identically zero. Therefore, there exists a disk $D=D\left(z_{0}, R\right)$, centered at some $z_{0} \in \Omega$ and with radius $R>0$, contained in $\Omega$ such that $\varphi^{\prime}(z) \neq 0$ for all $z \in D$. The forthcoming analysis is done in this disk.

Taking the Laplacian of both sides of (18), we obtain

$$
\left|\psi^{\prime}\right|^{2}=\left|\varphi^{\prime}\right|^{2}+\left|\omega_{1}^{\prime}\right|^{2}
$$

Since $\varphi^{\prime} \neq 0$, we get

$$
1+\left|\frac{\omega_{1}^{\prime}}{\varphi^{\prime}}\right|^{2}=\left|\frac{\psi^{\prime}}{\varphi^{\prime}}\right|^{2} .
$$

By Lemma 1 we see that both $\psi^{\prime} / \varphi^{\prime}$ and $\omega_{1}^{\prime} / \varphi^{\prime}$ are constant functions in $D$ and hence (by the identity principle) throughout $\Omega$. Let $m=\omega_{1}^{\prime} / \varphi^{\prime}$. Therefore [using also (19)] we have

$$
\omega_{1}^{\prime}=m \varphi^{\prime} \quad \text { and } \quad \psi^{\prime}=e^{i \theta} \sqrt{1+|m|^{2}} \varphi^{\prime},
$$

where $\theta$ is a real number. Thus, for certain complex constants $n$ and $p$,

$$
\omega_{1}=m \varphi+n \text { and } \psi=e^{i \theta} \sqrt{1+|m|^{2}} \varphi+p
$$

Using this information in (18), we get

$$
\begin{aligned}
1 & +\left(1+|m|^{2}\right)|\varphi|^{2}+|p|^{2}+2 \operatorname{Re}\left\{e^{i \theta} \bar{p} \sqrt{1+|m|^{2}} \varphi\right\} \\
& =|\varphi|^{2}+|m|^{2}|\varphi|^{2}+|n|^{2}+2 \operatorname{Re}\{m \bar{n} \varphi\} .
\end{aligned}
$$

Hence

$$
2 \operatorname{Re}\left\{\left(e^{i \theta} \bar{p} \sqrt{1+|m|^{2}}-m \bar{n}\right) \varphi\right\}=|n|^{2}-|p|^{2}-1
$$

As a consequence, we see that unless $e^{i \theta} \bar{p} \sqrt{1+|m|^{2}}-m \bar{n}=0$, the values of $\varphi$ lie on a line. This is not possible for non-constant $\varphi$. Therefore, we have

$$
e^{i \theta} \bar{p} \sqrt{1+|m|^{2}}=m \bar{n}
$$

and also

$$
|n|^{2}=1+|p|^{2}
$$

Taking absolute values in (21) gives $|p|^{2}\left(1+|m|^{2}\right)=|m|^{2}|n|^{2}$, while from (22) we have $|m|^{2}\left(1+|p|^{2}\right)=|m|^{2}|n|^{2}$. Therefore

$$
p=e^{i s} m
$$


for some real number $s$. Note that if $m=0$, then $\omega_{1}$ is constant, which contradicts our assumptions. Thus, $m \neq 0$ and from (21) we get

$$
n=\frac{e^{-i \theta} e^{i s} m \sqrt{1+|m|^{2}}}{\bar{m}}
$$

Expressing $\omega_{1}$ and $\varphi$ in the first relation in (20), we obtain

$$
\frac{G_{1}^{\prime}}{F_{1}^{\prime}}=m \frac{F_{2}^{\prime}}{F_{1}^{\prime}}+n
$$

Hence

$$
G_{1}^{\prime}=m F_{2}^{\prime}+n F_{1}^{\prime}
$$

and

$$
F_{2}^{\prime}=-\frac{n}{m} F_{1}^{\prime}+\frac{1}{m} G_{1}^{\prime}=-\frac{e^{-i \theta} e^{i s} \sqrt{1+|m|^{2}}}{\bar{m}} F_{1}^{\prime}+\frac{1}{m} G_{1}^{\prime} .
$$

Making $\psi, \varphi$ and $p$ explicit in the second relation in (20) gives

$$
\psi=\frac{G_{2}^{\prime}}{F_{1}^{\prime}}=e^{i \theta} \sqrt{1+|m|^{2}} \frac{F_{2}^{\prime}}{F_{1}^{\prime}}+e^{i s} m
$$

Multiplying this relation by $F_{1}^{\prime}$ and using (23), we deduce

$$
\begin{aligned}
G_{2}^{\prime} & =e^{i \theta} \sqrt{1+|m|^{2}} F_{2}^{\prime}+e^{i s} m F_{1}^{\prime} \\
& =e^{i \theta} \sqrt{1+|m|^{2}}\left(-\frac{e^{-i \theta} e^{i s} \sqrt{1+|m|^{2}}}{\bar{m}} F_{1}^{\prime}+\frac{1}{m} G_{1}^{\prime}\right)+e^{i s} m F_{1}^{\prime} \\
& =-\frac{e^{i s}}{\bar{m}} F_{1}^{\prime}+\frac{e^{i \theta} \sqrt{1+|m|^{2}}}{m} G_{1}^{\prime} .
\end{aligned}
$$

Finally, setting $\xi=s+\pi$ and denoting

$$
\alpha:=\frac{e^{i \xi} e^{-i \theta} \sqrt{1+|m|^{2}}}{\bar{m}}, \quad \beta:=\frac{e^{-i \xi}}{m}
$$

leads to

$$
\begin{aligned}
F_{2}^{\prime} & =\alpha F_{1}^{\prime}+e^{i \xi} \beta G_{1}^{\prime}, \\
G_{2}^{\prime} & =\bar{\beta} F_{1}^{\prime}+e^{i \xi} \bar{\alpha} G_{1}^{\prime},
\end{aligned}
$$

which is a re-expression of (17). 
Remark 2 Assume that $F_{1}+\overline{G_{1}}$ and $F_{2}+\overline{G_{2}}$ are two sense preserving harmonic mappings in the simply connected domain $\Omega$, related by (17). In the case when $F_{1}+\overline{G_{1}}$ is univalent and $\xi=0$, then $F_{2}+\overline{G_{2}}$ is obtained by composing with a sense preserving affine transformation. Since this affine transformation preserves univalence, $F_{2}+\overline{G_{2}}$ is univalent as well. This is not true if $\xi \neq 0$. For example, the so-called harmonic Koebe function introduced in [5] (see also [8, Sect. 5.3]), defined by $K=f+\bar{g}$, where

$$
f(z)=\frac{z-\frac{1}{2} z^{2}+\frac{1}{6} z^{3}}{(1-z)^{3}} \text { and } g(z)=\frac{\frac{1}{2} z^{2}+\frac{1}{6} z^{3}}{(1-z)^{3}}, \quad z \in \mathbb{D}
$$

is univalent in the unit disk $\mathbb{D}$ but there exists $|\mu|=1$ such that $f+\overline{\mu g}$ is not univalent (see [13, Theorem 7.1]).

\section{The solutions}

In what follows we will set

$$
F^{\prime}(t, z)=f(t, z) \text { and } G^{\prime}(t, z)=g(t, z)
$$

and use the notation $F_{0}+\overline{G_{0}}$ for the function $F(0, \cdot)+\overline{G(0, \cdot)}$, which is supposed to be univalent in the simply connected domain $\Omega_{0}$. Also, we write $f_{0}=f(0, \cdot)$ and $g_{0}=g(0, \cdot)$.

\subsection{Solutions in the linearly dependent case}

We start by finding the solutions $f \not \equiv 0$ and $g \not \equiv 0$ such that the governing Eq. (13) holds under the additional assumption that the initial harmonic (sense preserving) labelling map $F_{0}+\overline{G_{0}}$ is such that $F_{0}^{\prime}$ and $G_{0}^{\prime}$ are linearly dependent, or, in other words, $F_{0}+\overline{G_{0}}$ has constant dilatation. Indeed, since the harmonic map $F_{0}+\overline{G_{0}}$ is sense preserving, the linear dependence translates into the fact that there exists a constant $\lambda \in \mathbb{C}$ with $|\lambda|<1$ such that $G_{0}^{\prime}=\lambda F_{0}^{\prime}$.

Theorem 3 Let $\Omega_{0} \subset \mathbb{C}$ be a simply connected domain. If the initial harmonic (univalent, sense preserving) labelling map $F_{0}+\overline{G_{0}}$ satisfies $G_{0}^{\prime}=\lambda F_{0}^{\prime}$ for some $\lambda \in \mathbb{C}$, then the particle motion (9) of a fluid flow, defined by means of (24), is given by

$$
\left\{\begin{array}{l}
f(t, z)=\sqrt{1-|\lambda|^{2}+|\beta(t)|^{2}} e^{i \int_{0}^{t} \frac{v_{0}+\operatorname{Im}\left\{\beta(s) \overline{\beta_{t}(s)}\right\}}{1-|\lambda|^{2}+|\beta(s)|^{2}} d s} F_{0}^{\prime}(z), \\
g(t, z)=\beta(t) F_{0}^{\prime}(z)
\end{array}\right.
$$

where $\beta:[0, \infty) \rightarrow \mathbb{C}$ is a $C^{1}$ function with $\beta(0)=\lambda$, and $v_{0} \in \mathbb{R}$ is an arbitrary constant.

Proof Recall that we are using the notation $f_{0}=F_{0}^{\prime}$. Since the Jacobian of the labelling map remains unchanged at all times $t$, we can apply Theorem 1 to deduce 
that $f(t, z)=\alpha(t) f_{0}(z)$ and $g(t, z)=\beta(t) f_{0}(z)$, where $|\alpha|^{2}-|\beta|^{2} \equiv 1-|\lambda|^{2}>0$. Using this in (13), we obtain

$$
f_{t} \bar{f}-g \bar{g}_{t}=\left(\alpha_{t} \bar{\alpha}-\beta \bar{\beta}_{t}\right)\left|f_{0}(z)\right|^{2}=i v(z, \bar{z}) .
$$

As $F_{0}+\overline{G_{0}}$ is a sense preserving mapping, we have $\left|f_{0}\right|>0$. Thus $\alpha_{t} \bar{\alpha}-\beta \bar{\beta}_{t}=i v_{0}$ for some constant $v_{0} \in \mathbb{R}$, and we are lead to the system

$$
\left\{\begin{array}{l}
\alpha_{t} \bar{\alpha}-\beta \bar{\beta}_{t}=i v_{0} \\
|\alpha|^{2}=|\beta|^{2}+c
\end{array}\right.
$$

where $c=1-|\lambda|^{2}>0$. The second equation above ensures that $|\alpha|>0$, which allows us to write $\alpha(t)=R(t) e^{i \varphi(t)}$ for appropriate $C^{1}$-functions $R:[0, \infty) \rightarrow(0, \infty)$ and $\varphi:[0, \infty) \rightarrow \mathbb{R}$ (see, for instance, [17, Theorem 2.24]). The system (26) written in polar coordinates becomes

$$
\left\{\begin{array}{l}
R_{t} R+i R^{2} \varphi_{t}-\beta \bar{\beta}_{t}=i v_{0} \\
R=\sqrt{|\beta|^{2}+c}
\end{array}\right.
$$

The first equation in (27) in conjunction with the time-differentiation of the second equation in (27) yields $R^{2} \varphi_{t}=v_{0}+\operatorname{Im}\left\{\beta \bar{\beta}_{t}\right\}$. Thus (26) reduces to

$$
\left\{\begin{array}{l}
\varphi(t)=\varphi(0)+\int_{0}^{t} \frac{\nu_{0}+\operatorname{Im}\left\{\beta(s) \bar{\beta}_{t}(s)\right\}}{|\beta(s)|^{2}+c} d s \\
R=\sqrt{|\beta|^{2}+c}
\end{array}\right.
$$

The initial conditions $f(0, \cdot)=f_{0}, g(0, \cdot)=g_{0}$ now give $\beta(0)=\lambda$ and $\varphi(0)=0$. Therefore we obtain (25).

Example 1 Kirchhoff's solution [16] is the particular case of (25) in which

$$
F_{0}^{\prime}=A e^{i k z}, \quad \beta(t) \equiv \lambda, \quad \text { and } \quad \nu_{0}=0,
$$

where $A$ and $k$ are non-zero real constants and $\lambda \in(0,1)$. The condition on the univalence of $F_{0}$ requires that $\Omega_{0}$ does not contain points $z$ and $w$ with

$$
\operatorname{Im}\{z\}=\operatorname{Im}\{w\} \text { and } \operatorname{Re}\{z\}=\operatorname{Re}\{w\}+\frac{2 m \pi}{k}
$$

for some integer $m$.

\subsection{The solutions in the linearly independent case}

Using again the notation $F_{0}+\overline{G_{0}}$ for the function $F(0, \cdot)+\overline{G(0, \cdot)}$ and keeping in mind that $F_{0}+\overline{G_{0}}$ is sense preserving univalent in the simply connected domain 
$\Omega_{0}$, we have that $\left|F_{0}^{\prime}\right|^{2}-\left|G_{0}^{\prime}\right|>0$ in $\Omega_{0}$. Also, as before, we set $f_{0}=f(0, \cdot)$ and $g_{0}=g(0, \cdot)$ (hence $\left|f_{0}\right|^{2}-\left|g_{0}\right|^{2} \neq 0$ ).

Now, we will consider solutions $f \not \equiv 0$ and $g \not \equiv 0$ such that (13) holds and such that $F_{0}+\overline{G_{0}}$ has non-constant dilatation.

Theorem 4 Let $\Omega_{0} \subset \mathbb{C}$ be a simply connected domain. Assume that the initial harmonic (univalent, sense preserving) labelling map $F_{0}+\overline{G_{0}}$ is such that $F_{0}^{\prime}$ and $G_{0}^{\prime}$ are linearly independent. The particle motion (9) of a fluid flow, defined by means of (24), is either described by

$$
\left\{\begin{array}{l}
f(t, z)=\sqrt{1+|\beta(t)|^{2}} e^{i \int_{0}^{t} \frac{v_{0}+\operatorname{Im}\left\{\beta_{t}(s) \overline{\beta(s)\}}\right.}{1+|\beta(s)|^{2}} d s} F_{0}^{\prime}(z)+\beta(t) G_{0}^{\prime}(z), \\
g(t, z)=\overline{\beta(t)} F_{0}^{\prime}(z)+\sqrt{1+|\beta(t)|^{2}} e^{-i \int_{0}^{t} \frac{v_{0}+\operatorname{Im}\left\{\beta_{t}(s) \overline{\beta(s)\}}\right.}{1+|\beta(s)|^{2}} d s} G_{0}^{\prime}(z),
\end{array}\right.
$$

where $\beta:[0, \infty) \rightarrow \mathbb{C}$ is a $C^{1}$ function and $\nu_{0} \in \mathbb{R}$, or by

$$
\left\{\begin{array}{l}
f(t, z)=e^{i v_{0} t} F_{0}^{\prime}(z) \\
g(t, z)=e^{i\left(\xi_{0}-v_{0}\right) t} G_{0}^{\prime}(z)
\end{array}\right.
$$

where $v_{0}$ is as above and $\xi_{0} \in \mathbb{R} \neq\{0\}$. Moreover, for the solutions (28), univalence at any time is ensured once it holds at time $t=0$. Univalence holds for the solutions (29) if and only if $F_{0}+\overline{\lambda G_{0}}$ is univalent for all $\lambda$ with $|\lambda|=1$.

Proof By Theorem 2, we know that there exist $C^{1}$ functions $\alpha, \beta:[0, \infty) \rightarrow \mathbb{C}$ with $|\alpha(t)|^{2}-|\beta(t)|^{2}=1$ for all $t \geq 0$ and $\xi:[0, \infty) \rightarrow \mathbb{R}$ such that

$$
\left\{\begin{array}{l}
f(t, z)=\alpha(t) f_{0}(z)+e^{i \xi(t)} \beta(t) g_{0}(z), \\
g(t, z)=\overline{\beta(t)} f_{0}(z)+e^{i \xi(t)} \frac{\alpha(t)}{\alpha} g_{0}(z)
\end{array}\right.
$$

Note that since $f(0, \cdot)=f_{0}, g(0, \cdot)=g_{0}$, and the dilatation of $F_{0}+\overline{G_{0}}$ is nonconstant, we have the initial conditions

$$
\alpha(0)=1, \quad \beta(0)=0, \quad \text { and } \xi(0)=0 .
$$

A straightforward calculation shows that

$$
\begin{aligned}
f_{t} \bar{f}-g \bar{g}_{t}= & \left(\alpha_{t} \bar{\alpha}-\beta_{t} \bar{\beta}\right)\left(\left|f_{0}\right|^{2}-\left|g_{0}\right|^{2}\right)+2 i \operatorname{Re}\left\{\xi_{t} \alpha \bar{\beta} e^{-i \xi} f_{0} \overline{g_{0}}\right\} \\
& +i \xi_{t}\left(|\alpha|^{2}+|\beta|^{2}\right)\left|g_{0}\right|^{2} \\
= & \left(\alpha_{t} \bar{\alpha}-\beta_{t} \bar{\beta}-i \xi_{t}|\alpha|^{2}\right)\left(\left|f_{0}\right|^{2}-\left|g_{0}\right|^{2}\right)+i \xi_{t}\left|\alpha f_{0}+\beta e^{i \xi} g_{0}\right|^{2}
\end{aligned}
$$


Now, by (13), we know that $f_{t} \bar{f}-g \bar{g}_{t}=i v(z, \bar{z})$. Since the function $\left|f_{0}\right|^{2}-\left|g_{0}\right|^{2}$ only depends on $z$ and $\bar{z}$ and is always different from zero, we obtain that

$$
\begin{aligned}
& \left(\alpha_{t}(t) \overline{\alpha(t)}-\beta_{t}(t) \overline{\beta(t)}-i \xi_{t}(t)|\alpha(t)|^{2}\right) \\
& \quad+i \frac{\xi_{t}(t)\left|\alpha(t) f_{0}(z)+\beta(t) e^{i \xi(t)} g_{0}(z)\right|^{2}}{\left|f_{0}(z)\right|^{2}-\left|g_{0}(z)\right|^{2}}=i \widetilde{v}(z, \bar{z}),
\end{aligned}
$$

where $\widetilde{v}=v /\left(\left(\left|f_{0}\right|^{2}-\left|g_{0}\right|\right)^{2}\right)$. Let us re-write the previous equation using that $\left|f_{0}\right|^{2}-$ $\left|g_{0}\right|^{2}=\left|f_{0}\right|^{2}\left(1-|\omega|^{2}\right)$, where $\omega=g_{0} / f_{0}$ is the dilatation of $F_{0}+\overline{G_{0}}$. Note that since we are assuming that $\omega$ is not constant, we have that there exists an open disk $D \subset \Omega_{0}$ such that $\omega^{\prime}(z) \neq 0$ for all $z \in \Omega_{0}$. From now on, we will assume that $z \in D$. Within these terms (33) becomes

$$
\begin{aligned}
& \left(\alpha_{t}(t) \overline{\alpha(t)}-\beta_{t}(t) \overline{\beta(t)}-i \xi_{t}(t)|\alpha(t)|^{2}\right) \\
& \quad+i \frac{\xi_{t}(t)\left|\alpha(t)+\beta(t) e^{i \xi(t)} \omega(z)\right|^{2}}{1-|\omega(z)|^{2}}=i \widetilde{v}(z, \bar{z})
\end{aligned}
$$

Taking derivatives with respect to $z$ in (34), we obtain that the function

$$
\begin{aligned}
& i \xi_{t}(t) \frac{\omega^{\prime}(z)}{\left(1-|\omega(z)|^{2}\right)^{2}}\left(\beta(t) e^{i \xi(t)}+\alpha(t) \overline{\omega(z)}\right)\left(\overline{\alpha(t)}+\overline{\beta(t)} e^{-i \xi(t)} \overline{\omega(z)}\right) \\
& =i \xi_{t}(t)\left(\overline{\alpha(t)} \beta(t) e^{i \xi(t)}+\left(|\alpha(t)|^{2}+|\beta(t)|^{2}\right) \overline{\omega(z)}+\alpha(t) \overline{\beta(t)} e^{-i \xi(t)} \overline{\omega(z)}{ }^{2}\right) \\
& \quad \times \frac{\omega^{\prime}(z)}{\left(1-|\omega(z)|^{2}\right)^{2}}
\end{aligned}
$$

only depends on $z$ and on $\bar{z}$. Hence, so does

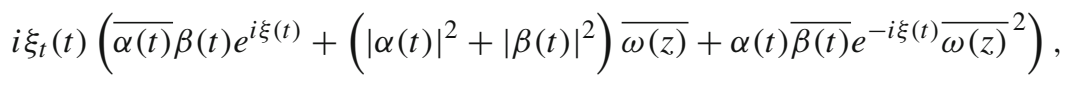

which has derivative with respect to $\bar{z}$ equal to

$$
i \xi_{t}(t)\left(|\alpha(t)|^{2}+|\beta(t)|^{2}\right) \overline{\omega^{\prime}(z)}+2 i \xi_{t}(t) \alpha(t) \overline{\beta(t)} e^{-i \xi(t) \overline{\omega(z) \omega^{\prime}(z)}}
$$

Since $\omega^{\prime}$ is supposed to be non-zero, we have that

$$
i \xi_{t}(t)\left(|\alpha(t)|^{2}+|\beta(t)|^{2}\right)+2 i \xi_{t}(t) \alpha(t) \overline{\beta(t)} e^{-i \xi(t)} \overline{\omega(z)}=\gamma(z, \bar{z})
$$

where $\gamma$ is a function of $z$ and $\bar{z}$. This fact finally implies (again taking derivatives with respect to $\bar{z}$ in (35) and dividing out by $\omega^{\prime}$ ) the equation

$$
\xi_{t} \alpha \bar{\beta} e^{-i \xi}=c_{1}
$$


for some constant $c_{1}$ and, in particular, by using this information in (35) we get that $\left(|\alpha|^{2}+|\beta|^{2}\right) \xi_{t}$ is constant as well. Moreover, by (32), we see that $\alpha \bar{\alpha}_{t}-\beta \bar{\beta}_{t}$ is constant too (indeed, using that $|\alpha|^{2}-|\beta|^{2}=1$, it is easy to check that $\alpha_{t} \bar{\alpha}-\beta_{t} \bar{\beta}$ is a purely imaginary complex number). In other words, we have seen that the following system must be satisfied by the functions $\alpha, \beta$, and $\xi$ (here, $c_{1}, c_{2}$, and $c_{3}$ are certain constants with $c_{3} \in \mathbb{R}$ ):

$$
\left\{\begin{array}{l}
\xi_{t} \alpha \bar{\beta} e^{-i \xi}=c_{1}, \\
\left(|\alpha|^{2}+|\beta|^{2}\right) \xi_{t}=c_{2}, \\
\alpha_{t} \bar{\alpha}-\beta_{t} \bar{\beta}=i c_{3}, \\
|\alpha|^{2}-|\beta|^{2}=1 .
\end{array}\right.
$$

Now, note that the second and fourth equations in (36) can be re-written as

$$
\left\{\begin{array}{l}
|\alpha|^{2} \xi_{t}+|\beta|^{2} \xi_{t}=c_{2} \\
|\alpha|^{2} \xi_{t}-|\beta|^{2} \xi_{t}=\xi_{t}
\end{array}\right.
$$

which gives

$$
\left\{\begin{array}{l}
2|\alpha|^{2} \xi_{t}=c_{2}+\xi_{t} \\
2|\beta|^{2} \xi_{t}=c_{2}-\xi_{t}
\end{array}\right.
$$

On the other hand, using the first equation in (36), a direct consequence of (37) is that

$$
4\left|c_{1}\right|^{2}=4\left(\xi_{t} \alpha \bar{\beta} e^{-i \xi}\right) \overline{\left(\xi_{t} \alpha \bar{\beta} e^{-i \xi}\right)}=2|\alpha|^{2} \xi_{t} \cdot 2|\beta|^{2} \xi_{t}=c_{2}^{2}-\xi_{t}^{2}
$$

This shows that $\xi_{t}^{2}$ (hence $\xi_{t}$ ) must be a constant function.

We distinguish between two types of solutions.

Case $1 \quad \xi_{t} \equiv 0$. Then $\xi$ must be constant and hence, by (31), we see that $\xi \equiv 0$. Moreover, in this case (36) becomes

$$
\left\{\begin{array}{l}
\alpha_{t} \bar{\alpha}-\beta_{t} \bar{\beta}=i c_{3} \\
|\alpha|^{2}-|\beta|^{2}=1
\end{array}\right.
$$

Notice that the above system is a particular case of (26), with $v_{0}=c_{3}, c=1$, and where $\beta$ is replaced by $\bar{\beta}$. Taking into account that $\alpha(0)=0$, the previous approach shows that its solution is given by

$$
\alpha(t)=\sqrt{|\beta(t)|^{2}+1} \exp \left(i \int_{0}^{t} \frac{c_{3}+\operatorname{Im}\left\{\beta_{t}(s) \overline{\beta(s)}\right\}}{1+|\beta(s)|^{2}} d s\right),
$$

and $\beta:[0, \infty) \rightarrow \mathbb{C}$ is an arbitrary $C^{1}$-function with $\beta(0)=0$. These considerations prove the first part of the theorem. The univalence is obtained as in Remark 2. 
Case $2 \xi_{t} \equiv \xi_{0}, \quad \xi_{0} \neq 0$. Note that in this case [using that, by $(31), \xi(0)=0$ ], we have $\xi(t)=\xi_{0} t$. By (37) we see that both functions $|\alpha|$ and $|\beta|$ are constants. A further application of (31) gives that $\beta \equiv 0$ and $\alpha(t)=e^{i \varphi(t)}$ for some $C^{1}$ function $\varphi:[0, \infty) \rightarrow \mathbb{R}$ with $\varphi(0)=0$. Moreover, by (36) we get $\varphi^{\prime} \equiv c_{3}$, so that $\varphi(t)=c_{3} t$. Therefore, according to (30), we have that

$$
\left(\begin{array}{l}
F^{\prime}(t, z) \\
G^{\prime}(t, z)
\end{array}\right)=\left(\begin{array}{ll}
1 & 0 \\
0 & e^{i \xi_{0} t}
\end{array}\right)\left(\begin{array}{ll}
e^{i c_{3} t} & 0 \\
0 & e^{-i c_{3} t}
\end{array}\right)\left(\begin{array}{l}
F_{0}^{\prime}(z) \\
G_{0}^{\prime}(z)
\end{array}\right)
$$

which is (29) with $v_{0}=c_{3}$. Concerning the issue of univalence for the flows (29), note that

$$
\left(\begin{array}{l}
F(t, z) \\
G(t, z)
\end{array}\right)=\left(\begin{array}{ll}
1 & 0 \\
0 & e^{i \xi_{0} t}
\end{array}\right)\left(\begin{array}{ll}
e^{i \nu_{0} t} & 0 \\
0 & e^{-i \nu_{0} t}
\end{array}\right)\left(\begin{array}{l}
F_{0}(z) \\
G_{0}(z)
\end{array}\right)+\left(\begin{array}{l}
\mu(t) \\
v(t)
\end{array}\right)
$$

where $\mu$ and $v$ are real-valued $C^{1}$ functions in $[0, \infty)$. That is,

$$
F(t, z)+\overline{G(t, z)}=e^{i \nu_{0} t}\left(F_{0}(z)+e^{-i \xi_{0} t} \overline{G_{0}(z)}\right)+v(t)
$$

where $v(t)$ is the translation vector $(\mu(t), \overline{v(t)})$. The translation vector $v$, as well as the multiplication factor $e^{i \nu_{0} t}$, play no role regarding univalence. Since $e^{-i \xi_{0} t}$ covers the unit circle as $t \in[0, \infty)$, the claim is proved.

Example 2 Gerstner's flow [9] corresponds to the case of (29) in which

$$
\nu_{0}=0, \quad \xi_{0}=\sqrt{k \mathfrak{g}}, \quad F_{0}^{\prime}=1, \quad \text { and } \quad G_{0}^{\prime}(z)=-e^{-i k z}
$$

where $k>0, \mathfrak{g}$ is the gravitational constant of acceleration, and $z \in \Omega_{0}=\{z \in$ $\mathbb{C}: \operatorname{Im}\{z\}<0\}$.

Acknowledgements This research was developed during the second author's research stay at the University of Vienna funded by the ERC Advanced Grant "Nonlinear studies of water flows with vorticity". She wants to use this opportunity to thank the faculty and staff members at the Faculty of Mathematics for their hospitality. Finally, we would like to thank the referees for their careful reading of the manuscript as well as for their useful suggestions.

Open Access This article is distributed under the terms of the Creative Commons Attribution 4.0 International License (http://creativecommons.org/licenses/by/4.0/), which permits unrestricted use, distribution, and reproduction in any medium, provided you give appropriate credit to the original author(s) and the source, provide a link to the Creative Commons license, and indicate if changes were made.

\section{References}

1. Abrashkin, A.A., Yakubovich, E.I.: Two-dimensional vortex flows of an ideal fluid. Dokl. Adad. Nauk SSSR 276, 76-68 (1984) [in Russian; English translation: Soviet Phys. Dokl. 29, 370-371 (1984)]

2. Aleman, A., Constantin, A.: Harmonic maps and ideal fluid flows. Arch. Ration. Mech. Anal. 204, 479-513 (2012)

3. Bennet, A.: Lagrangian fluid dynamics. Cambridge University Press, Cambridge (2006) 
4. Chuaqui, M., Duren, P., Osgood, B.: The Schwarzian derivative for harmonic mappings. J. Anal. Math. 91, 329-351 (2003)

5. Clunie, J., Sheil-Small, T.: Harmonic univalent functions. Ann. Acad. Sci. Fenn. Ser. A. 9, 3-25 (1984)

6. Constantin, A.: Edge waves along a sloping beach. J. Phys. A. 34, 9723-9731 (2001)

7. Constantin, A.: An exact solution for equatorially trapped waves. J. Geophys. Res. 117, C05029 (2012). doi:10.1029/2012JC007879

8. Duren, P.L.: Harmonic mappings in the plane, Cambridge Tracts in Mathematics, vol. 156. Cambridge University Press, Cambridge (2004)

9. Gerstner, F.: Theorie der Wellen samt einer daraus abgeleiteten Theorie der Deichprofile. Ann. Phys. 2, 412-445 (1809)

10. Godin, O.A.: Finite-amplitude acoustic-gravity waves: exact solutions. J. Fluid Mech. 767, 52-64 (2015)

11. Henry, D.: An exact solution for equatorial geophysical water waves with an underlying current. Eur. J. Mech. B Fluids 38, 18-21 (2013)

12. Henry, D., Hsu, H.-C.: Instability of equatorial water waves in the $f$-plane. Discrete Contin. Dyn. Syst. 35, 909-916 (2015)

13. Hernández, R., Martín, M.J.: Stable geometric properties of analytic and harmonic functions. Math. Proc. Camb. Philos. Soc. 155, 343-359 (2013)

14. Hernández, R., Martín, M.J.: Pre-Schwarzian and Schwarzian derivatives of harmonic mappings. J. Geom. Anal. 25, 64-91 (2015)

15. Ionescu-Kruse, D.: Instability of edge waves along a sloping beach. J. Differ. Equations 256, 3999-4012 (2014)

16. Kirchhoff, G.: Vorlesungen über matematische Physik. Mechanik Teubner, Leipzig (1876)

17. Kühnel, W.: Differential geometry. curves-surfaces-manifolds. Translated from the 1999 German original by Bruce Hunt. Student Mathematical Library, vol. 16. American Mathematical Society, Providence (2002)

18. Leblanc, S.: Local stability of Gerstner's waves. J. Fluid Mech. 506, 245-254 (2004)

19. Rankine, W.J.M.: On the exact form of waves near the surface of deep water. Philos. Trans. R. Soc. Lond. Ser. A 153, 127-138 (1863) 